\title{
MONITORAMENTO E CONTROLE DE DESGASTE DO REFRATÁRIO PARA OPERAÇÕES CONTÍNUAS DE ALTO- FORNO*
}

\section{Resumo}

\author{
Afshin Sadri2 \\ Sergey Filatov ${ }^{1}$ \\ Ivan Kurunov ${ }^{1}$ \\ Yakov Gordon ${ }^{2}$ \\ Wai Lai Ying ${ }^{2}$ \\ Jennifer Erskine ${ }^{2}$
}

Uma das principais razões para o reparo de altos-fornos é a perda do revestimento refratário, principalmente nas regiões da soleira e onde são feitos os furos de corrida. A operação intensa dos altos-fornos pode resultar em desgaste e deteorização extensiva do material refratário. Isto pode, então, afetar a continuidade das operações e a vida útil do forno. Novo-Lipetsk (NLMK) e a Hatch desenvolveram uma abordagem sistemática para monitorar a condição do revestimento da soleira dos altos-fornos usando Acousto Ultrasonic-Eco (AU-E), um sistema de ensaios nãodestrutivos desenvolvido pela Hatch. Inspeções contínuas e periódicas dos altosfornos revelaram áreas com deterioração acelerada do material refratário, formação extensa de "elefant foot" e acelerado desgaste do refratário, assim como fissuras e outras anomalias. Para prolongar a vida do forno, mantendo a intensidade de suas operações, algumas modificações, tais como a melhoria da qualidade do coque e da limpeza das placas de resfriamento e adição de óxido de titânio, entre outras medidas, foram recomendadas e aplicadas. Este trabalho demonstra como esforços coordenados entre a NLMK e a Hatch resultaram no prolongamento da vida útil de um alto-forno

Palavras-chave: Monitoramento e inspeção de alto-forno; Ensaio não destrutivo ("Non-Destructive Testing", NDT); Desgaste de refratário; Vida útil do alto-forno.

\section{MONITORING AND CONTROL OF REFRACTORY WEAR FOR INTENSIVE OPERATION OF BLAST FURNACE}

\begin{abstract}
One of the main reasons for blast furnace reline is the loss of refractory lining, particularly in the hearth and taphole regions. Intense operation of the blast furnace can result in extensive refractory wear and deterioration. This can then affect the continuity of the operation and the furnace campaign life. Novo-Lipetsk (NLMK) and Hatch developed a systematic approach to monitor the condition of blast furnace hearth linings using Acousto Ultrasonic-Echo (AU-E), a non-destructive testing system developed by Hatch. Continuous and periodic inspections of blast furnaces revealed areas with accelerated refractory deterioration, formation of elephant foot, extent and speed of refractory wear, as well as cracks and other anomalies. Improvement in coke quality, stave washing, the addition of titania, and other changes were recommended and implemented to prolong furnace life while maintaining the intensity of furnace operation. This paper demonstrates how the coordinated efforts between NLMK and Hatch resulted in prolonging the blast furnace campaign life.
\end{abstract}

Keywords: Blast furnace inspection and monitoring; Non-Destructive Testing (NDT); Refractory deterioration; Blast furnace campaign.

Novo-Lipetsk Metallurgical Combine (NLMK), Lipetsk, Lipetsk Oblast, Rússia.

Hatch Ltd, Mississauga, Ontario, Canada. 


\section{INTRODUÇÃO}

A operação intensiva de um alto-forno (AF) oferece muitos benefícios, como aumento na produção de metal quente e da rentabilidade das operações de ferro e aço. Entretanto, a operação intensiva também pode aumentar a taxa de desgaste do refratário comprometendo a integridade estrutural do alto-forno, especialmente se não forem tomadas medidas para proteger o revestimento refratário e a geração de um acréscimo ("build-up") de forma estável. Este artigo descreve como o Ensaio Não-Destrutivo (NDT) foi utilizado em altos-fornos NLMK para monitorar e reduzir o desgaste do refratário na soleira, mantendo a operação intensiva.

A Novo-Lipetsk Metallurgical Combine (NLMK) é um gigante russo do setor siderúrgico, produzindo anualmente 12,7 milhões de toneladas de aço. A planta tem 5 altos-fornos operacionais: os seus parâmetros de projeto e de operação estão apresentados na Tabela 1

Tabela 1. Parâmetros de projeto e de operação dos altos-fornos NLMK

\begin{tabular}{|c|c|c|c|c|c|}
\hline Parâmetros & AF 3 & $\mathrm{AF} 4$ & $\mathrm{AF} 5$ & AF 6 & $\mathrm{AF} 7$ \\
\hline Último grande reparo, mês, ano & $\begin{array}{l}\text { Set. } \\
2000\end{array}$ & $\begin{array}{c}\text { Mai } \\
1999\end{array}$ & $\begin{array}{c}\text { Dezembro } 1996 \\
\text { (Abril } 2015- \\
\text { paredes da soleira) }\end{array}$ & $\begin{array}{l}\text { Julho } \\
1993\end{array}$ & $\begin{array}{l}\text { Sopro - em } \\
\text { Agosto } \\
2011\end{array}$ \\
\hline $\begin{array}{l}\text { Volume de trabalho do forno ( "working } \\
\text { volume", WV), } \mathrm{m}^{3}\end{array}$ & 1,700 & 1,700 & 2,760 & 2,760 & 3,561 \\
\hline Diâmetro da soleira, m & 10 & 10 & 12 & 12 & 13.1 \\
\hline Carga metalica Fetotal (fluxo), \% & 57.26 & 57.93 & 56.86 & 58.75 & 57.18 \\
\hline Taxa de coque, $\mathrm{kg} / \mathrm{thm}$ & 422.8 & 302.7 & 321.3 & 395.2 & 390 \\
\hline Taxa de PCl, kg/thym & & 134.7 & 120.6 & & \\
\hline Taxa de gás natutral, $\mathrm{Nm}^{3} / \mathrm{thm}$ & 98.3 & 53.1 & 63.1 & 128.2 & 129.9 \\
\hline Adição de oxigênio, \%, no forno & 27.48 & 28.13 & 27.1 & 30.22 & 30.86 \\
\hline Temperatura de sopro, ${ }^{\circ} \mathrm{C}$ & 1,100 & 1,139 & 1,096 & 1,174 & 1.222 \\
\hline Produção diaria, thm/day & 4,765 & 5,622 & 8,177 & 8,284 & 12,500 \\
\hline $\begin{array}{l}\text { Produtividade específica, thm } / \mathrm{m}^{3} / \text { day } \\
\text { (WV) }\end{array}$ & 2.8 & 3.31 & 2.99 & 3.03 & 3.51 \\
\hline $\begin{array}{l}\text { Produtividade específica, thm } / \mathrm{m}^{2} / \text { day }(\varnothing \\
\text { soleira) }\end{array}$ & 60.7 & 71.62 & 72.36 & 73.3 & 92.8 \\
\hline Taxa de escória, kg/thm & 389 & 361 & 378 & 333 & 380 \\
\hline
\end{tabular}

A Tabela 1 mostra que os altos-fornos da NLMK operaram comparativamente com baixo teor de ferro total na carga metálica, com valores no intervalo de $56,86 \%$ $58,75 \%$. Além disso, os fornos operam com altos níveis de adição de oxigênio $(27,2 \%$ - 30,86\%), taxas moderadas de adição de coque e combustíveis suplementares, e temperaturas acima de 1100 oC. Apesar do baixo teor de ferro total na carga metálica e de um elevado volume de escória, o processo de redução é bastante intenso com uma produtividade específica variando de $60,7 \mathrm{thm} / \mathrm{m} 2 /$ dia $(2,8$ thm/m3/dia) para o AF \# 3 e 92,8 thm $/ \mathrm{m} 2 /$ dia $(3,51$ thm $/ \mathrm{m} 3 /$ dia) para o AF \# 7 .

Dos 5 altos-fornos, o mais antigo em serviço é forno \# 6, com um tempo de operação de 23 anos e o mais novo (vida útil de 5 anos) é o forno \# 7. O tempo de operação dos altos-fornos \# 3, 4 e 5 são de 16, 17 e 20 anos, respectivamente. A operação contínua dos altos-fornos, especialmente dos mais velhos (\# 3-6), exige um acompanhamento cuidadoso das condições de revestimento refratário na soleira do forno. Esse monitoramento permite que medidas preventivas sejam tomadas para retardar o desgaste do refratário e criar um acréscimo de proteção estável nas paredes da soleira, impedindo o ataque químico e térmico pelo metal quente. 
Os altos-fornos \# 5, 6 e 7 são equipados com termopares incorporados. Fornos \# 6 e 7 têm modelos térmicos para estimar o perfil do revestimento remanescente (espessura do refratário além do "build-up"). No entanto, ao longo do tempo diversos termopares foram danificados, deixando poucos ou nenhum termopar em determinadas áreas. Os altos-fornos \# 3 e 4 estão sem termopares. Esta falta de informação faz com que a estimativa de desgaste do refratário e da extensão do "build-up" seja difícil.

Em 2013 a NLMK contratou a Hatch para realizar testes não-destrutivos nos altosfornos da NLMK usando a tecnologia Acousto Ultrasonic - Echo (AU-E) patenteada pela Hatch [1]. A NLMK e a Hatch desenvolveram uma abordagem sistemática para monitorar a condição dos revestimentos das soleiras dos altos-fornos. Esta abordagem envolve inspeções trimestrais e semestrais dos altos-fornos mais velhos (\#5 e 6) e do mais novo BF (\# 7). Além disso, foram realizadas inspeções anuais nas soleiras dos outros altos-fornos. Os resultados de cada inspeção foram cuidadosamente discutidos com os gestores e operadores das plantas, de forma que medidas posteriores foram implementadas para retardar ou parar a deterioração do refratário e prolongar a vida útil dos altos-fornos.

\section{MÉTODO AU-E DE ENSAIO NÃO DESTRUTIVO EM REFRATÁRIO}

Acousto Ultrasonic Echo (AU-E) é uma técnica de propagação de ondas de tensão capaz de medir a espessura das camadas de refratários e de "build-up" de material. AU-E usa análise de dados de tempo e frequência para determinar a espessura do refratário e detectar anomalias, tais como fissuras, lacunas ou a intrusão de metal dentro do revestimento refratário. Durante a medição, um impacto mecânico sobre a superfície da estrutura gera um pulso de tensão, que se propaga para as camadas refratárias. A onda é refletida parcialmente pela mudança nas propriedades do material de cada camada do revestimento refratário, mas também se propaga através das camadas refratárias sólidas atavés das interfaces tijolo/tijolo, tijolo/gás, ou tijolo/metal fundido. As ondas de compressão (ou ondas-p) são coletadas por um receptor e os sinais são analisados para avaliação da qualidade e espessura do refratário.

Os principais detalhes do método AU-E estão apresentados nesse trabalho. Uma descrição mais detalhada do método AU-E é apresentada em vários outros trabalhos [2-4]. 

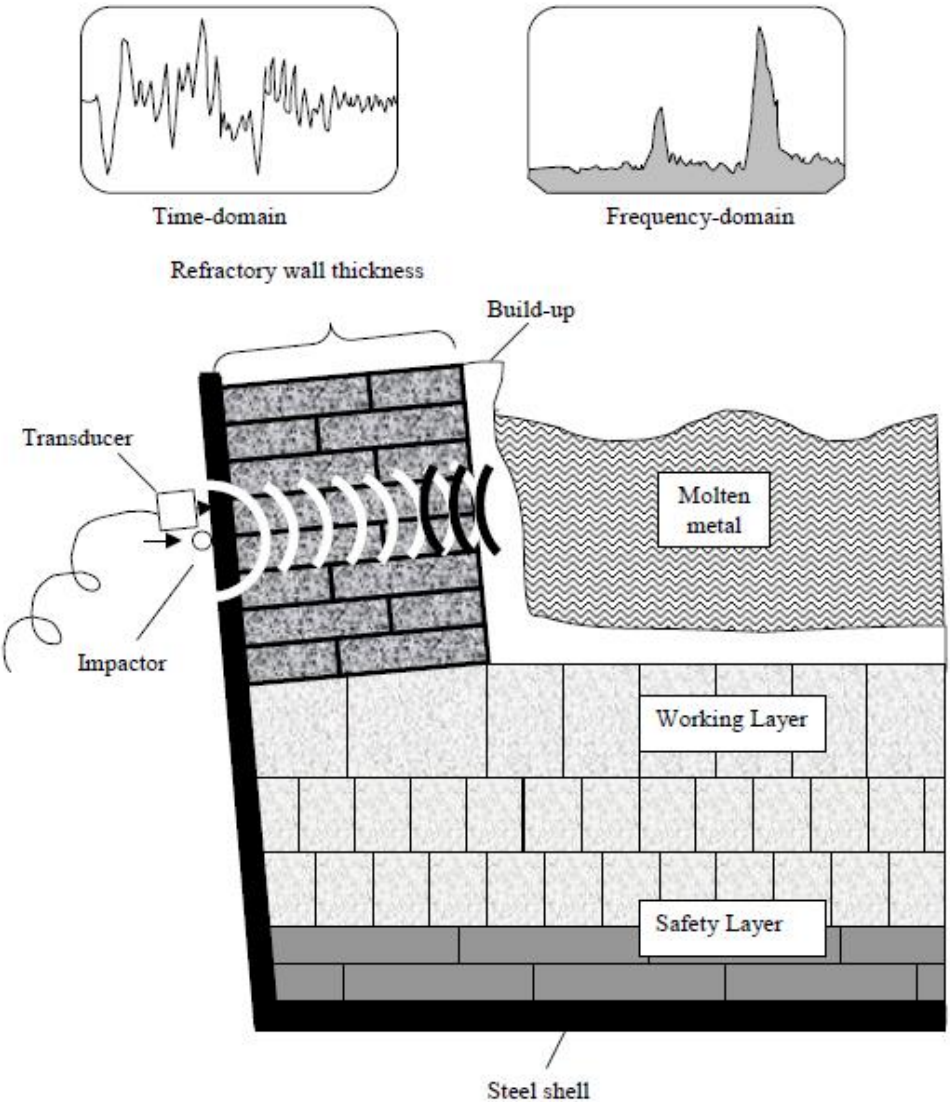

Figura 1. llustração do método AU-E .

Os dados de campo recolhidos no domínio do tempo são extremamente complexos, contendo numerosas frequências e múltiplas reflexões, difrações e refrações das ondas superficiais e internas. Convertidos para o domínio da frequência, os resultados são muito melhor definidos. Uma menor frequência de reflexão corresponde a uma maior distância da interface do sinal refletido. Os picos distintos na amplitude do sinal correspondem aos limites entre o metal líquido e o "build-up", "build-up" e refratário e os locais de anomalias (fissuras, carbono oxidado etc.) A primeira amplitude distinguível à frequência mais baixa está associada aos limites entre o "build-up" e o metal quente, a segunda amplitude distinguível à maior frequência caracteriza o limite entre o "build-up" e o refratário, e a terceira amplitude distinguível à mais alta frequência mostra a posição de uma fissura ou anomalia.

A espessura da camada de material, utilizando a técnica AU-E, é estimada pela equação seguinte:

$$
T=\frac{\alpha \beta V_{p}}{2 f_{p}}
$$

Onde T é a espessura ou profundidade da superfície refletora; $f_{p}$ é a frequência da onda-P; $\mathrm{V}_{\mathrm{p}}$ é a velocidade de propagação da onda-P no material; a é o fator de correção da temperatura; $\beta$ é o fator de forma.

$O$ fator de forma $\beta$, contribui para a redução na velocidade devida a formas variadas de fornos, tais como cilíndrico ou retangular. Para altos-fornos onde as dimensões laterais são, pelo menos, seis vezes a espessura do revestimento, o fator $\beta$ é 0.96 . O fator de correção térmica, a é a razão entre o módulo de elasticidade, em condições de operação $\left(E_{x}\right)$ e o módulo de elasticidade do refratário à temperatura 
ambiente $\left(E_{0}\right)$ : $\alpha=E_{x} / E_{0}$. Na maioria dos casos assume-se que o módulo de elasticidade de Young do refratário muda linearmente entre a face quente e a fria em função da temperatura.

Para uma seção múltipla de camadas, tais como na soleira, a espessura da camada final de refratário $\left(T_{n}\right)$ é calculada com base na seguinte equação:

$$
T_{n}=\frac{\left(V_{p}\right)_{n} \alpha_{n} \beta_{n}}{2}\left[\frac{1}{f}-\sum_{i=1}^{n-1} \frac{2 T_{i}}{\left(V_{P}\right)_{i} \alpha_{i} \beta_{i}}\right]
$$

onde $\mathrm{n}$ refere-se a camada específica do revestimento da soleira; $f$ é a frequência de ressonância para a espessura da camada de ordem enésima; i refere-se a espessura inicial da camada do refratário.

Antes da coelta de dados de campo, a velocidade aparente da onda-P de cada camada de tijolo é determinada por calibração de amostras representativas de tijolo à temperatura ambiente. As medições de calibração de velocidade da onda devem ser efetuadas em todos os materiais através dos quais a onda se propagar. $O$ fator $\alpha$ pode ser calculado experimentalmente, pelo aquecimento de amostras de tijolos e medindo as velocidades de onda nas temperaturas desejadas, ou pode ser calculada pelas propriedades elásticas e térmicas do tijolo em particular.

Se amostras do tijolo não estiverem disponíveis (por exemplo, no caso da NLMK) a Hatch utiliza as propriedades de tijolos semelhantes a partir de catálogos de fornecedor ou medidas realizadas em projetos anteriores para estimar a velocidade aparente da onda-P. Esta aproximação gera algumas incertezas na estimativa da exatidão da espessura do refratário, o que é uma parte integrante do erro total de medição de 4-7\%. A estimativa da espessura do "build-up" é menos precisa (erro é de aproximadamente 15\%), uma vez que as propriedades do "build-up" raramente são conhecidas e podem variar substancialmente.

\section{EXEMPLOS DE MEDIDAS}

A partir de julho de 2013, as medições AU-E do refratário residual foram realizadas a cada seis meses para soleiras dos altos-fornos \#5 e 6. Estes dois fornos foram escolhidos devido a suas condições e idade (20 e 23 anos em operação para os altos fornos \# 5 e 6, respectivamente). Os altos-fornos \# 5 e 6 produzem, em conjunto, aproximadamente $41-42 \%$ das mais de 37 mil toneladas totais de metal quente produzidos por dia pela NLMK. Portanto, a operação estável desses dois fornos é fundamental para o sucesso de todo o ramo siderúrgico da empresa.

Os resultados AU-E para alto-forno \#5 estão apresentados na Figura 2. Ela mostra o teste semestral em novembro de 2013 e Julho de 2014. O perfil estimado do refratário restante em julho 2014 é mostrado em azul. O "build-up" detectado está mostrado em verde, e as anomalias são mostradas em vermelho. Anomalias podendo ser representadas por rachaduras ou delaminações dentro do refratário, ou refratário solto. Para efeito de comparação, o perfil do refratário restante em Novembro é mostrado em amarelo, mostrando claramente as áreas onde ocorreu o desgaste do refratário. 


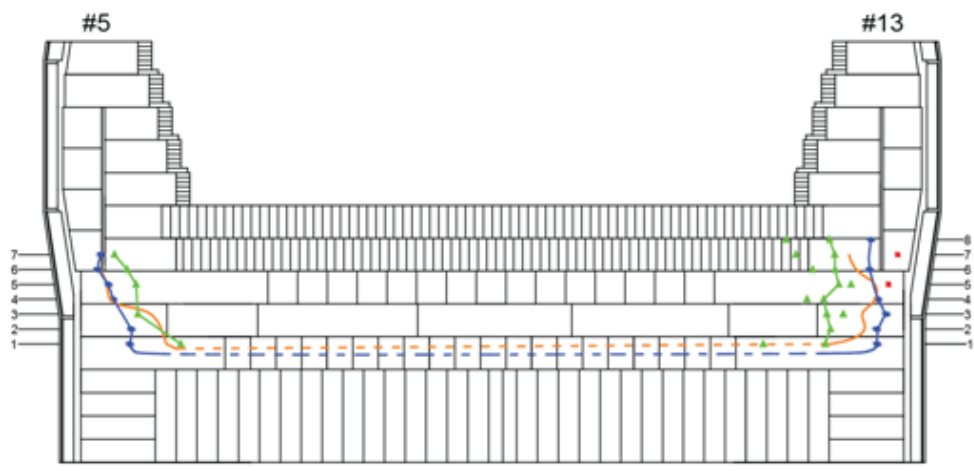

Legend

July 2014

- Detected Refractory Thickness

- Detected Crack or Loose Castable Location

4 Detected Accretion Thickness

— July 2014Estimated Remaining Refractory Thickness

- Estimated Accretion Thickness

Nov 2013

- Estimated Remaining Refractory Thickness

Figura 2. Resultados dos ensaios de AU-E na soleira do AF \#5 (Novembro de 2013 e Julho de 2014).

Os resultados dos testes mostraram aumento significativo de desgaste das paredes da soleira baixa ao longo do tempo e a formação de "elephant foot ". A formação de "elephant foot " no cadinho do alto-forno pode ser atribuída às condições do "homem morto", que constitui o fluxo de metal na região inferior do forno. O "homem morto" deve ser flutuante e permeável para o metal quente evite o fluxo periférico excessivo de alta velocidade do metal quente e a erosão do revestimento refratário.

De acordo com a Figura 2 e os resultados do AU-E, em Julho de foram feitas as seguintes conclusões para o AF\#5:

- As primeiras duas placas de fundo da soleira foram desgastadas. A terceira placa de fundo foi parcialmente desgastada.

- A média do refratário remanescente foi de $700 \mathrm{~mm}$ em Julho de 2014 (média da espessura do refratário foi de $1710 \mathrm{~mm}$ ). Isso equivale a $41 \%$ da espessura do refratário restante. Ocorreu uma redução de $7 \%$ da espessura residual de refratário desde Novembro de 2013 (espessura restante de 48\%).

A Figura 3 mostra os resultados mais recentes do alto-forno \#5. Os resultados correspondem à última inspeção de Fevereiro de 2015 antes de parada do forno para reparos na soleira. O perfil estimado do refratário restante em Fevereiro de 2015 está mostrado em azul, enquanto o perfil de Julho de 2014 é mostrado em amarelo. Os dois perfis são muito semelhantes se comparados com os perfis mostrados na Figura 2, o que indica que o desgaste do refratário ocorreu de maneira mais estável entre Julho de 2014 e Fevereiro de 2015.

De acordo com a Figura 3 e os resultados do AU-E, em Fevereiro de 2015 de foram feitas as seguintes conclusões para o AF\#5:

- A média do refratário remanescente foi de $41 \%$ da espessura média original de $1710 \mathrm{~mm}$. Esta observação é similar à da investigação feita em Julho de 2014 (espessura restante de $41 \%$ ).

- O resultado do NDT atual indicou que o TiO2 foi efetivamente afetado, e produziu uma espessura de "build-up" substancial na face quente do refratário. A extensão média de "build-up" é de cerca de $1440 \mathrm{~mm}$ medidos a partir face fria do refratário, que é semelhante ao levantamento anterior, em Julho de 2014 (média de 1460 milímetros). 

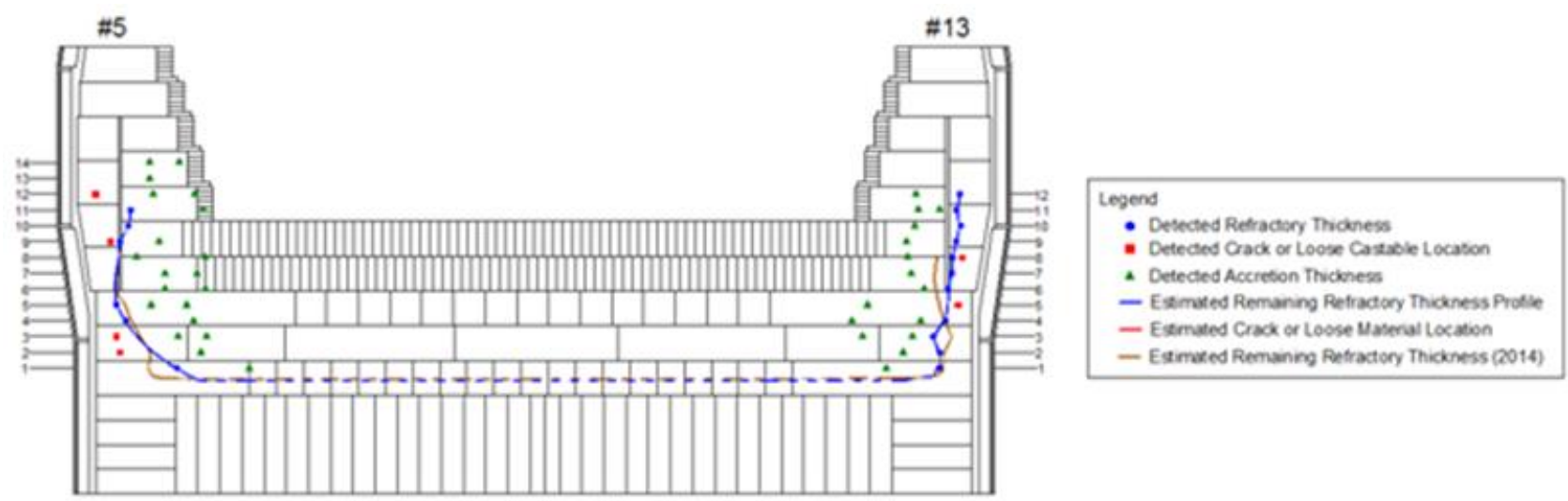

Figure 3. Resultados dos ensaios de AU-E na soleira do AF \#5 (Fevereiro de 2015)

O alto-forno \#7 "Rossiyanka" foi testado em duas oportunidades, em Fevereiro e Setembro de 2015. Os resultados do teste para esse forno são apresentados na Figura 4.
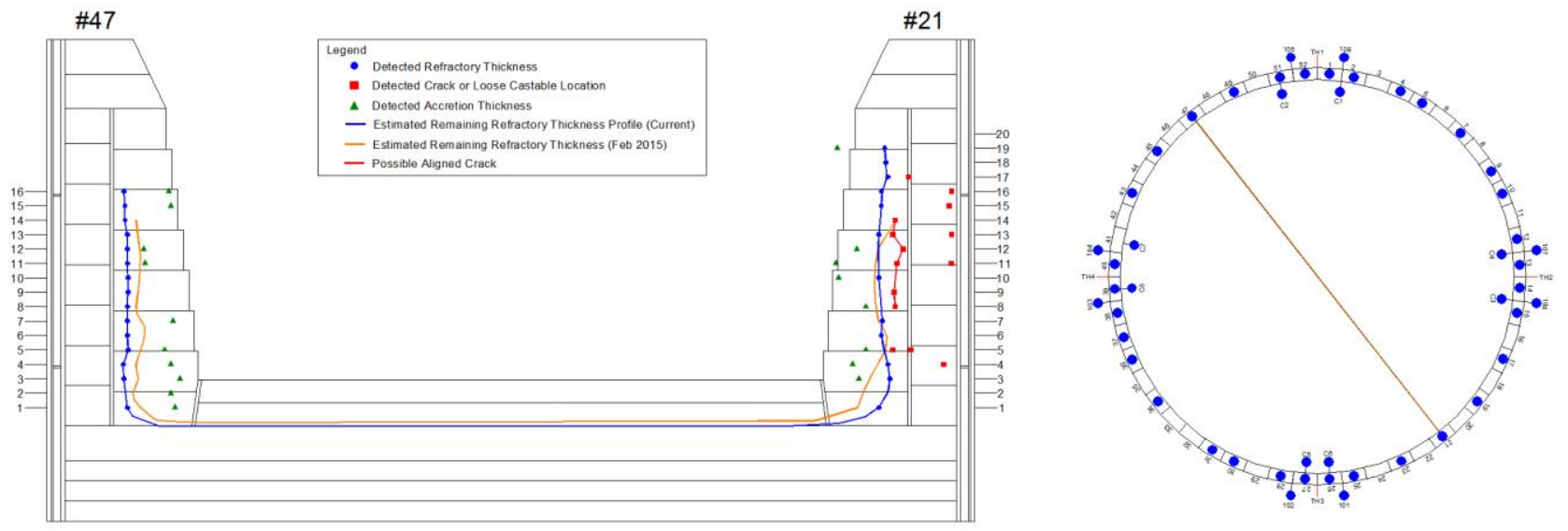

Figura 4. Resultados de AU-E para o alto-forno \#7 (Setembro de 2015)

Os resultantes das medições AU-E no alto-forno \#7 levaram as seguintes conclusões:

- As duas primeiras camadas de blocos de carbono no fundo do forno foram desgastadas

- A espessura média do refratário médio remanescente das paredes da soleira é $1130 \mathrm{~mm}, 51 \%$ da espessura média original de $2147 \mathrm{~mm}$. Isto demonstra um desgaste médio de 13\% desde Fevereiro de 2015 (1410 milímetros, 64\% restante). A maior parte do desgaste ocorreu perto da região dos furos de corrida. Ao se afastar desta região, o desgaste é significativamente menor.

- A espessura mínima detectada do refratário restante é de 850 a $870 \mathrm{~mm}$. Estes pontos correspondem a um refractário remanescente de $38 \%$ a $40 \%$.

- Os resultados dos testes mostram o início da formação do "elephant foot" no cadinho do AF7.

- "build-up"s estáveis de 300 to $1,000 \mathrm{~mm}$ de espessura foram formados ao longo do revestimento do refratário da soleira protegendo este de intenso desgaste. 
Os resultados AU-E do alto-forno \#7 mostram que durante os quatro anos e meio de funcionamento, as paredes laterais de soleira de alto-forno perderam em média de quase $50 \%$ de sua espessura de refratário inicial. A perda intensiva de revestimento refratário deste forno provavelmente aconteceu durante os primeiros dois anos e meio de operação, quando o coque CSR estava na faixa de $45-55 \%$. O forno operou com nível de "homem morto" e soleira cheios, o que levou a operação não-estável e perda de produtividade. A partir de 2014, a qualidade do coque CSR foi melhorando gradualmente para a faixa de 62 a $64 \%$, o que melhorou o desempenho do forno e condições do "homem morto". A adição de shungite e titânio criou um "build-up" estável nas paredes da soleira que protege o revestimento refratário de uma maior deterioração rápida. O alto-forno pode continuar sua operação intensivamente, enquanto um acompanhamento de NDT anual das condições da soleira e da região de furos de corrida são necessários, para as medidas preventivas em tempo hábil.

\section{PRECISÃO DA ESTIMATIVA DA ESPESSURA DO REFRATÁRIO}

Houve a parada e resfriamento do alto-forno \#5 em Março de 2015 para instalação de um novo revestimento de soleira. Isso proporcionou uma oportunidade para verificar a precisão das medições AU-E. Para fazer uma comparação direta, a Hatch realizou medições uma semana antes do desligamento desse alto-forno. Foi realizada uma perfuração para amostragem enquanto o forno ainda estava quente. Depois que o forno foi drenado e arrefecido, foram feitas medições físicas com fita da espessura restante. Estas medidas foram realizadas pelo pessoal de alto-forno.

O furo para a sondagem foi feito no nível 12, enquanto que as medições físicas foram tomadas no nível 8 (Figura 5). Estes resultados foram comparados com as correspondentes medidas AU-E com grande semelhança.

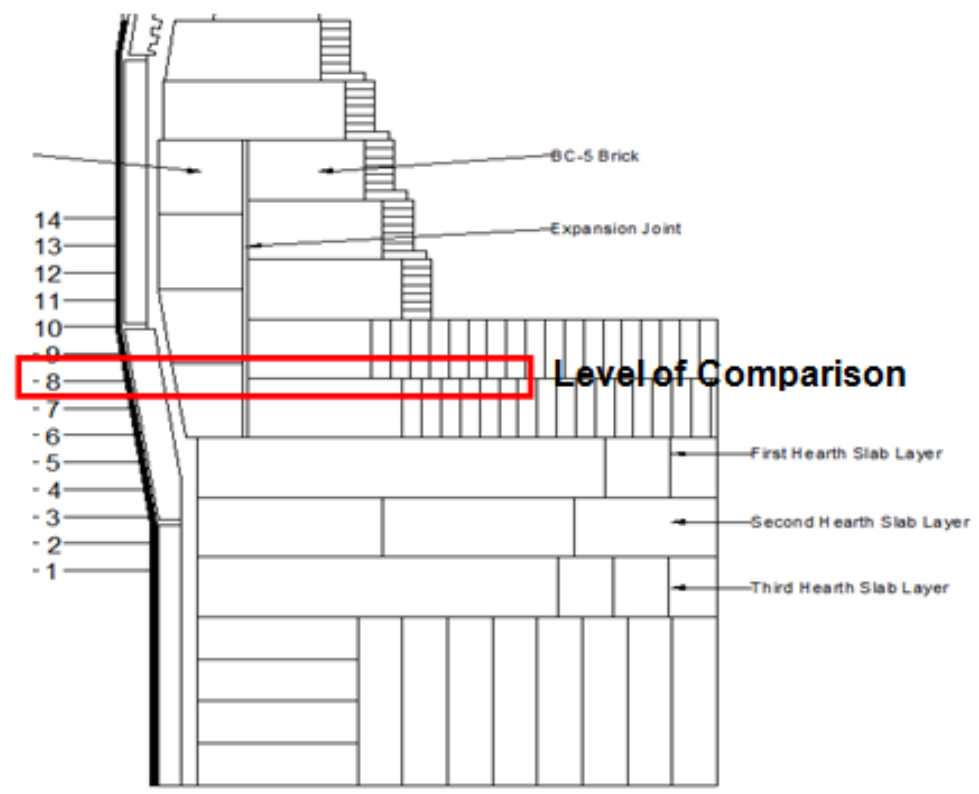

Figura 5. Localização do furo para sondagem e das medições fisicas.

O resultado da sondagem revelou uma espessura remanescente de refratário de $530 \mathrm{~mm}$, enquanto medições AU-E indicam uma espessura refratário de $500 \mathrm{~mm}$. Isto representa uma diferença de $6 \%$. A comparação dos resultados $A U-E$ com os resultados das medições com a fita são apresentados na Tabela 2: 
Tabela 1. Precisão das medidas de AU-E

\begin{tabular}{|c|c|c|c|c|c|c|}
\hline \multirow{2}{*}{ Linha } & \multirow{2}{*}{ Level } & \multicolumn{2}{|c|}{$\begin{array}{c}\text { Medição de Refratário } \\
\text { Remanescente em Fevereiro de } 2015 \\
(\mathrm{~mm})\end{array}$} & \multirow{2}{*}{$\begin{array}{l}\text { Diferença, } \\
(+/-\mathrm{mm})\end{array}$} & \multirow{2}{*}{$\begin{array}{l}\text { Diferença, } \\
\% \text { do AU- } \\
\text { E }\end{array}$} & \multirow[t]{2}{*}{ Observações } \\
\hline & & $\begin{array}{l}\text { Medição } \\
\text { Hatch AU-E }\end{array}$ & $\begin{array}{l}\text { Medição Fisica com } \\
\text { FIta }\end{array}$ & & & \\
\hline 3 & 8 & 550 & 530 & 20 & 4 & Boa Correlação \\
\hline 6 & 8 & 780 & 750 & 30 & 4 & Boa Correlação \\
\hline 9 & 8 & 650 & 600 & 50 & 8 & Boa Correlação \\
\hline 11 & 8 & 700 & 720 & 20 & 3 & Boa Correlação \\
\hline 15 & 8 & 570 & 520 & 50 & 9 & Boa Correlação \\
\hline \multicolumn{2}{|l|}{ Média } & 652 & 607 & 45 & 7 & \\
\hline
\end{tabular}

As diferenças entre as medições físicas e as medições AU-E variam de $20-50 \mathrm{~mm}$. A comparação dos resultados AU-E e as medições físicas (Tabela 2) confirmaram que a precisão de AU-E é de cerca de 4 a $7 \%$. O erro devido à aproximação das propriedades refratárias está incluso dentro desta faixa.

\section{EXTENSÃO DA VIDA ÚTIL DO ALTO-FORNO}

A Hatch e a NLMK propuseram várias estratégias para retardar o desgaste do refratário e prolongar a campanha de forno e a NLMK, em seguida, implementou essas medidas. Com inspeções regulares de NDT, o estado do refratário foi avaliado a cada 6 meses. Isto permitiu que os efeitos dessa campanha de medição se estenda a medidas, monitoramento e ajustes conforme necessário, levando a uma estabilização do desgaste refratário em ambos os altos-fornos \# 5 e 6.

As medidas implementadas para retardar o desgaste refratário são as seguintes:

- A adição de shungite à carga metálica na quantidade de $35-37 \mathrm{~kg} / \mathrm{thm}$. Shungite é um mineral natural com depósitos conhecidos apenas em Carélia, Rússia, que consiste em 58-62\% de $\mathrm{SiO} 2$ e $28-32 \%$ de carbono [5]. A temperaturas da ordem de $1400{ }^{\circ} \mathrm{C}$, sílica e carbono da shungite começam a reagir formando carboneto de silício ( $\mathrm{SiC})$. SiC reage parcialmente com os produtos fundidos e penetra parcialmente no interior da soleira na forma sólida e precipita nas paredes do alto-forno formando um "build-up" semelhante ao de carbonetos e nitritos de titânio.

- A adição de titânio em quantidades de 7-12 kg TiO2/thm. TiO2 forma carboneto de titânio e nitritos de titânio, que se precipitam sobre uma parede de soleira.

- A melhoria da qualidade do coque CSR de $45-55 \%$ para $63-65 \%$ e redução do CRI - de 27-30\% para 23-24\%. Esta melhoria permitiu um aumento da permeabilidade do "homem morto" e uma diminuição da velocidade circunferencial do metal quente, que promove a formação de "elephant foot".

- A limpeza das placas de resfriamento permite a remoção da tubulação de água melhorando assim a eficiência da transferência de calor, o que ajuda na formação de um "build-up" estável.

- Formação de barreiras ("tongues") abaixo dos furos de corrida usando uma grande quantidade de refratário projetado para reduzir a velocidade periférica de metal quente no forno, etc.

Todas estas medidas adotadas permitiram a continuação das corridas de alto-forno, mantendo as operações contínuas. 
De acordo com as pesquisas de NDT, as tendências de desgaste dos refratários nos altos fornos \# 5 e 6 são apresentadas na Figura 6 e Figura 7.

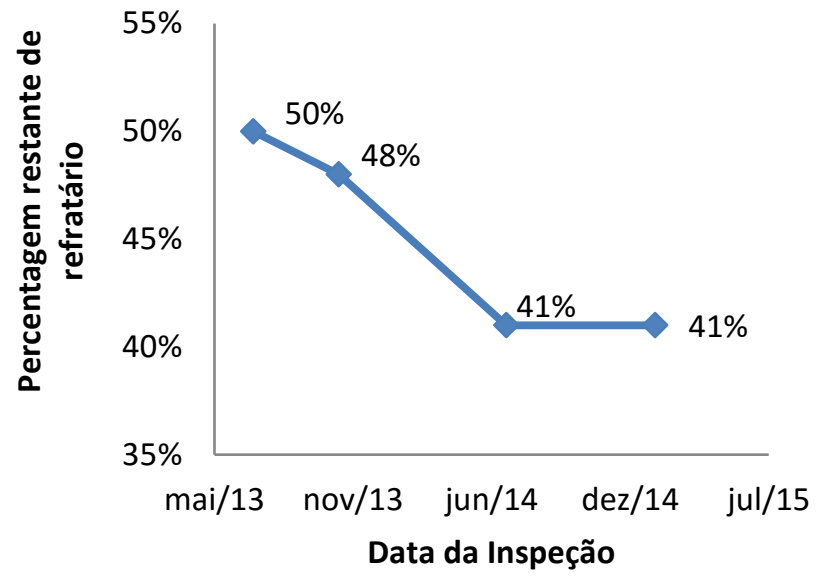

Figura 6. Tendências de desgaste médio do alto-forno \#5.

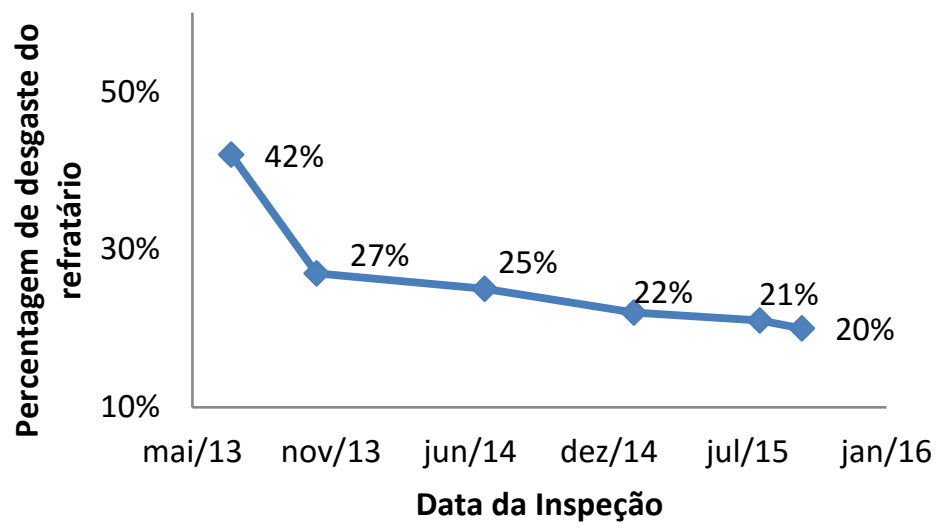

Figure 7. Tendências de desgaste médio do alto-forno \#6.

Após o início do teste AU-E em 2013, o desgaste médio do refratário entre Maio de 2013 e Julho de 2014 foi de 9\% para o alto-forno \#5 e 17\% para alto-forno \#6. De Julho de 2014 até Fevereiro de 2015, a espessura média do revestimento refratário na soleira do alto-forno \#5 foi estabilizado em um refratário remanescente com $41 \%$ da espessura original. A estabilização do desgaste refratário no alto-forno \#6 teve início em Novembro de 2013 com a adição regular de shungite e titânio. Note-se que os efeitos da adição shungite sobre a formação de "build-up" foram descobertos pela primeira vez no AF \#6 em 2002-2004 durante os ensaios para a substituição parcial de coque por shungite. Similar ao alto-forno \#7, a qualidade do coque no alto-forno \#6 também foi melhorada a partir de 2013. Para alto-forno \#6 a taxa média de desgaste do refratário variou entre os quatro últimos períodos de medição, variando de $0.5 \% /$ mês para $0.17 \% /$ mês.

\section{ESTIMATIVA DE ALERTA DE TEMPERATURA}

A combinação dos resultados de AU-E com as medições de temperatura por termopares (geralmente mais próximos da face fria dos refratários) permite uma estimativa do alerta de temperatura. Este alerta de temperatura indica que o revestimento refratário atingiu a espessura mínima aceitável, por exemplo, 30\% do 
revestimento inicial (incluindo acreção). Supõe-se que o fluxo de calor a partir de metal quente para o "build-up" solidificado através da parede continua a ser o mesmo e que o coeficiente de condutividade é um valor médio ponderado da condutividade dos refratários e do "build-up".

O método de estimativa para o alerta de temperatura é apresentado na Figura 8 . 0 caso 1 é o cenário atual e o caso 2 é o caso de predição quando há $30 \%$ de espessura do revestimento restante. Aqui $S_{n}$ é a localização de termopares a partir da interface do encaixe da placa de resfriamento; $S$ é a espessura restante atualmente; S30 representa $30 \%$ da espessura remanescente; T é a temperatura do termopar; T1' é a temperatura do primeiro termopar primeiro é atingido $30 \%$ do revestimento remanescente; TBS é a temperatura da interface do encaixe da placa de resfriamento e $1150^{\circ} \mathrm{C}$ é a temperatura de solidificação do metal quente.

A equação final para a estimativa de alerta de temperatura é a seguinte:

$$
\mathrm{T}^{\prime}{ }^{\prime}=1150-\left[(1150-\mathrm{T} 1)^{*}(\mathrm{~S} 30-\mathrm{S} 1)\right] /(\mathrm{S}-\mathrm{S} 1)
$$

Os resultados da inspeção e leituras AU-E dos termopares em Novembro 2013 foram usadas para estimar os resultados apresentados na Tabela 2. A espessura de refratário mais próxima do termopar medido pelo metódo AU-E foi usada nos cálculos. Com base na literatura, assumiu-se que o erro do termopar também está no intervalo de de $20-25 \%$. Naturalmente, tudo isto contribui para o cálculo dos erros.

A Tabela 2 mostra que a estimativa da espessura do refratário restante utilizando 0 fluxo de calor e a espessura do refratário remanescente medido por AU-E diferem em 3-15\%. O T1' estimado (quando restam 30\% do refratário) é pelo menos duas vezes o T1 medido nos pontos correspondentes. Isto indicou que esta zona do forno ainda ainda é operado em segurança.
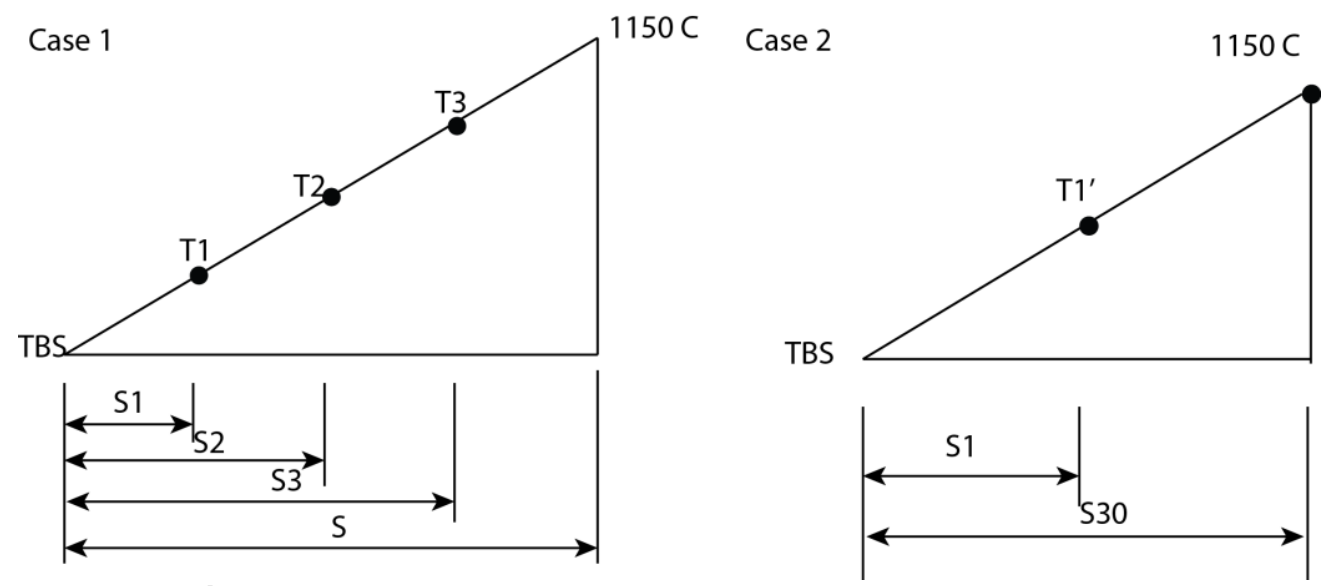

Figura 8. Esquema para a estimativa do alerta de temperatura

Tabela 2. Exemplo para a estimativa do alerta de temperatura

\begin{tabular}{|c|c|c|c|c|c|c|c|}
\hline \multirow[b]{3}{*}{$\mathrm{T} 1\left({ }^{\circ} \mathrm{C}\right)$} & \multirow[b]{3}{*}{$\mathrm{T} 2\left({ }^{\circ} \mathrm{C}\right)$} & \multirow[b]{3}{*}{$\mathrm{T} 3\left({ }^{\circ} \mathrm{C}\right)$} & \multirow{2}{*}{\multicolumn{2}{|c|}{ Ponto de Referência }} & \multirow{3}{*}{$\begin{array}{l}\text { Estimativa de } \\
\text { temperatura do } \\
\text { refratário restante, } \\
\mathrm{mm}\end{array}$} & \multirow{3}{*}{$\begin{array}{l}\text { AU-E Medição } \\
\text { do Refratário } \\
\text { Restante }(\mathrm{mm})\end{array}$} & \multirow[b]{3}{*}{$\mathrm{T} 1^{\prime}\left({ }^{\circ} \mathrm{C}\right)$} \\
\hline & & & & & & & \\
\hline & & & $\begin{array}{l}\text { Corte da } \\
\text { Seção } \\
\text { Vertical }\end{array}$ & Ponto & & & \\
\hline 331 & 528 & 781 & 11 & 9 & 669 & 710 & 681 \\
\hline 271 & 433 & 572 & 2 & 9 & 964 & 820 & 729 \\
\hline 327 & 512 & - & 10 & 12 & 817 & 840 & 649 \\
\hline
\end{tabular}




\section{CONCLUSÕES}

As operações contínuas de alto-forno requerem um controle cuidadoso das condições do revestimento do refratário da soleira. Os estudos de casos nos altosfornos da NLMK mostram que a AU-E é uma tecnologia confiável de estimativa da espessura dos refratários, e "build-up"s de localização de fissuras ou anomalia com uma precisão de 4-7\%. A aplicação da tecnologia AU-E para altos-fornos NLMK revelou as condições do revestimento refratário, incluindo a formação de "build-up" e as regiões de maior desgaste. Isto permitiu a NLMK e a Hatch desenvolverem e implementarem medidas preventivas para prolongar a vida útil e realizar a operação do forno de maneira segura. Estas medidas incluem (mas não estão limitadas a) a adição de shungite, materiais de óxido de titânio, limpeza das placas de resfriamento e utilização de coque de qualidade mais elevada. Todos estes permitem que a NLMK controle cuidadosamente as condições de alto-forno, mantendo a sua operação contínua.

\section{Agradecimentos}

Um agradecimento especial para nossos colegas da Novo-Lipetsk Metallurgical Combine, Rússia e Hatch, Canadá por sua valiosa contribuição com seu trabalho na realização deste trabalho.

\section{REFERENCIAS}

1 Sadri, A. (2006). Systems, methods and apparatus for non-disruptive and nondestructive inspection of metallurgical furnaces and similar vessels: patent application publication: US 20060186585.

2 Sansalone, M.J., \& Streett W.B. (1997). Impact-Echo: Nondestructive Evaluation of Concrete and Masonry, Bullbrier Press, Ithaca, NY. p. 339.

3 Sadri, A. (2005, August). Non-destructive determination of refractory and build-up thickness in operating furnaces using an acousto ultrasonic reflection technique. Proceedings of the Materials Degradation: Innovation, Inspection, Control and Rehabilitation Symposium, COM2005. Calgary, Alberta, Canada.

4 Sadri, A., Gordon, I., \& Rampersad, A. (2007, May). Acousto ultrasonic-echo (AU-E): A non-destructive testing technique for blast furnace hearth refractory condition monitoring., AdMet 2007, Proceedings of International Conference Advances in Metallurgical Processes and Materials, Vol. 2. 77-85, Dnipropetrovsk, Ukraine.

5 Kurunov, I., Lyapin, S., Titov. V., Loginov, V. and Polyakov N. (2006, November). New technological solutions for the lining protection of the blast furnace hearth. Proceedings of the 4th International congress on Science and Technology of Ironmaking (ICSTI'06), Osaka, Japan, November 26-30, 2006, p.p.543-546. 